\title{
A Four-Layer Odd Function Symmetrical Dual-Polarization Equilateral Right Triangle Slot Printed Broadband Directional Antenna for Wireless Lab Measurement Applications
}

\author{
Chang-Ju Wu, ${ }^{1}$ I-Fong Chen $\mathbb{D}^{\mathrm{D}},{ }^{2}$ Jwo-Shiun Sun, ${ }^{1}$ Chia-Mei Peng, ${ }^{3}$ Kang-Ling Li, ${ }^{2}$ \\ and Chung-Hao Huang ${ }^{2}$ \\ ${ }^{1}$ Department of Electronic Engineering, National Taipei University of Technology, No. 1, Sec. 3, Zhongxiao E. Road, \\ Taipei 10608, Taiwan \\ ${ }^{2}$ School of Electronics and Electrical Engineering, Dongguan Polytechnic/Department of Computer and Communication, \\ Jinwen University of Science and Technology, No. 99, An-Chung Road, Hsin-Tien District, New Taipei City 23154, Taiwan \\ ${ }^{3}$ Department of Communication Engineering, Feng Chia University, No. 100 Wenhwa Road, Seatwen District, \\ Taichung 40724, Taiwan
}

Correspondence should be addressed to I-Fong Chen; ex0206@just.edu.tw

Received 15 November 2020; Accepted 3 May 2021; Published 12 May 2021

Academic Editor: Muhammad Zubair

Copyright (C) 2021 Chang-Ju Wu et al. This is an open access article distributed under the Creative Commons Attribution License, which permits unrestricted use, distribution, and reproduction in any medium, provided the original work is properly cited.

A four-layer odd function symmetrical dual-polarization coplanar waveguide- (CPW-) fed equilateral right triangle slot printed broadband directional antenna for wireless lab measurement applications is presented. The proposed antenna consists of two stacked perpendicular odd function symmetrical equilateral right triangle slot antenna structures with two stacked reflector ground planes. It is miniaturized, simple, and easy to be fabricated. The measured results demonstrate that the proposed antenna can achieve wide impedance bandwidth that is almost $520 \%$ for $\left|S_{11}\right|$ and $\left|S_{22}\right|$; VSWR $\leqq 3$ which has implemented the operating band from 1.37 to $7.12 \mathrm{GHz}$ for Global Positioning System (GPS, $1.575 \mathrm{GHz}$ ), Wireless Local Area Networks band (2.4 GHz and $5.8 \mathrm{GHz}), 6 \mathrm{GHz}$ unlicensed spectrum $(5.925 \mathrm{GHz} 7.125 \mathrm{GHz})$, Long Term Evolution Upper Band (LTE, 1710-2690 MHz), and sub- $65 \mathrm{G}$ band $(3.5 \mathrm{GHz}$ and $4.7 \mathrm{GHz})$ applications. The measured in-band isolation performance between the two input ports $\left|S_{12}\right| \leqq-17 \mathrm{~dB}$ is significantly achieved, and meanwhile, the antenna radiation pattern, peak gain, and efficiency of the proposed antenna are measured as well. In the end, the radiation pattern data are compared and analyzed with simulation results.

\section{Introduction}

With the explosive development of the mobile and wireless communication industry in recent years, the wireless systems supporting wider bandwidth have become more and more popular and challenging. The demand to enhance the information accessibility with higher user experienced data throughput, low latency, and wideband utility has become more challenging in mobile and wireless technology, especially for highly integrated mobile and wireless system performance measurements. To fulfill the demand and the convenience of system measurement, wideband and multiband antennas are widely utilized in mobile and wireless communication systems to cover multiple communication bands, such as mobile wireless communication system (2 G/ $3 \mathrm{G} / 4 \mathrm{G}$ ) bands operating at $698-960 \mathrm{MHz} / 1710-2690 \mathrm{MHz}$, wireless local area network (WLAN) bands operating at 2.4-2.484 GHz/5.15-5.825 GHz, $6 \mathrm{GHz}$ unlicensed spectrum for the European markets between $5.925 \mathrm{GHz}$ and $6.425 \mathrm{GHz}$ and for the US markets between $5.925 \mathrm{GHz}$ and $7.125 \mathrm{GHz}$, and sub- $6 \mathrm{GHz} 5 \mathrm{G}$ bands operating at $3.5 \mathrm{GHz} /$ $4.7 \mathrm{GHz}$. In order to efficiently increase the measurement capability of the wireless lab, the device under test (DUT) link antenna is better to have a compact size, low cost, wideband, and dual-polarization so this kind of antenna structure is the primary consideration in our research. In [1], 
the first approach of the tripolarization antenna was presented for operating band $2.4 \mathrm{GHz}$ to $2.6 \mathrm{GHz}$, but the proposed antenna of the band coverage and isolation between some ports were not sufficient and needed to be improved in multiband wireless performance measurement applications. A few papers [2, 3] have been published for getting better isolation in some of the dual-polarization antenna applications, but the two proposed antennas of the impedance bandwidth are still not wide enough to cover modern mobile and wireless communication systems. For the purpose of a wider operating bandwidth and simple planar antenna configuration, bow-tie dipole and slot antenna structure are good candidates in reference research [4-7]. In paper [8], a dual-polarization coplanar waveguide(CPW-) fed bow-tie slot antenna has been demonstrated, the antenna structure is simple, and the specifications of wide bandwidth and high isolation can be accomplished easily. The final approach is for directional antenna with dualpolarization design consideration and application. In these literatures, the antennae have the features of directional, broadband, and dual-polarization which are presented. But, the operating bandwidth is not enough to cover the wider bandwidth, and the applications scenario is just limited for mobile base station [9-13]. To summarize all of the reference papers as above, we propose a new design for high gain, high isolation, and broadband directional antenna, which is comprised of the equilateral right triangle slot with odd function symmetrical pair of stubs; there are four stacked layers composed of two antennae and two reflectors are shown. The horizontal and vertical polarization are determined by the direction of the CPW-fed line, and the antenna is perpendicular to the top and bottom. Details of the specification of the proposed antenna designs and the experimental results of constructed prototypes are presented and discussed.

\section{Antenna Structure and Design}

In the beginning, we studied the antenna structure in-depth in the paper $[4,8]$ and simulated using Ansys High Frequency Structure Simulator (HFSS) to verify the antenna wideband performance. We have observed that the lowest operating frequency can be calculated by the following equation:

$$
f_{L}=\frac{c}{\lambda_{L}} \cong \frac{C}{\text { slot length }} \times 0.8(\mathrm{GHz}),
$$

where $C$ is the speed of light and Slot length represents the equilateral right triangle slot edge total length.

Further investigating the antenna structure, we understand that the structure is comprised of two symmetric bowtie slot structures that can be associated with the even and odd functions. In mathematics, even function and odd function are functions that satisfy particular symmetry relations, and therefore, we follow the reasonable deliberation to further carry out the constructive research of this paper. The top view of the basic geometry of the proposed antenna is shown in Figure 1. The antenna structure consists of an odd function symmetrical CPW-fed equilateral right triangle slot with an overall dimension of $100 \times 100 \mathrm{~mm}^{2}$. The

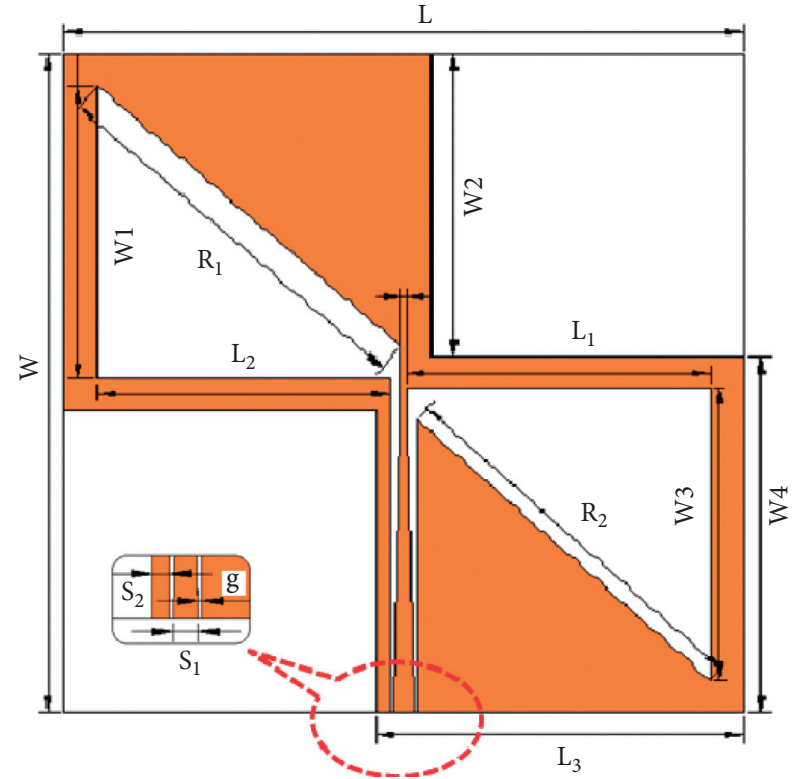

FIGURE 1: Geometry and dimension of the proposed odd function symmetrical equilateral right triangle slot broadband antenna.

antenna pattern is printed with a thickness of $1.6 \mathrm{~mm} \mathrm{FR} 4$ glass epoxy substrate (the relative permittivity is 4.4 ) and the loss tangent is 0.02 . The radiating element section of two equilateral right triangle slot opposite length is defined as L1/ L2, hypotenuse length is defined as R2/R1, and adjacent length is defined as $\mathrm{W} 3 / \mathrm{W} 1$. The fundamental resonant mode of modified equilateral right triangle slot structure is designed for the lowest operating band $1.5 \mathrm{GHz}$ and the length and width of the slots can be determined from the full, half, and quarter-wave lengths for each of the resonant frequencies. The antenna dimension of $\mathrm{L}$ and $\mathrm{W}$ is approximately $0.5 \lambda(=100 \mathrm{~mm})$, which can be determined by the lowest frequency of the desired band. The antenna of each right triangle slot edge with its total length of $0.75 \lambda$ $(\mathrm{L} 1+\mathrm{R} 2+\mathrm{W} 3 \approx 147 \mathrm{~mm})$ is designed for covering broadband operation, and it can be determined by the lowest frequency of the desired band. The CPW-fed is one of the best antenna structures to obtain a wider impedance matching, so the $\mathrm{CPW}$-fed line is designed to be $50 \Omega(\mathrm{S} 1=3 \mathrm{~mm})$, the gap distance $(g=0.45 \mathrm{~mm})$, taper to the CPW of signal strip length should be $0.25 \lambda$ (W2 $=46 \mathrm{~mm}$ ), and the parameters are determined by the lowest frequency of the desired band as well. The final optimized values of each parameter are listed in Table 1, and the comparison table of the relationship between the wavelength and the equilateral right triangle slot path length is shown in Table 2.

The comparison of the simulated current distribution of the proposed antenna $\{(\mathrm{R} 1+\mathrm{W} 1+\mathrm{L} 2):(\mathrm{R} 2+\mathrm{W} 3+\mathrm{L} 1)=1: 1)\}$ corresponds to the equilateral right triangle slot length to prove how the wide bandwidth performance works, and the antenna body is shown in Figure 2. At $1.575 \mathrm{GHz}, \lambda_{\mathrm{L}}$ can be approximately calculated through equation (1) and the relationship of the length can be considered as L1/L2 which is equivalent to a quarter wavelength; the result is compared with the total length of the triangular slot on the lowest frequency $1.575 \mathrm{GHz}$ of the desired band. At $2.4 \mathrm{GHz}$, the 
TABLE 1: Dimensions of the proposed odd function symmetrical equilateral right triangle slot broadband antenna.

\begin{tabular}{lccccccc}
\hline Parameter & L & L1 & L2 & L3 & S1 & S2 & g \\
\hline Value $(\mathrm{mm})$ & 100 & 44.5 & 43.05 & 54 & W1 & W2 & W3 \\
Parameter & R1 & R2 & W & W & W4 \\
Value $(\mathrm{mm})$ & 59.42 & 58.43 & 100 & 44.38 & 46 & 44.5 & 54 \\
\hline
\end{tabular}

TABLE 2: Comparison table of wavelength and relative equilateral right triangle slot path length.

\begin{tabular}{|c|c|c|c|c|c|}
\hline Frequency $(\mathrm{GHz})$ & $\lambda=\left(c / \sqrt{\varepsilon_{\mathrm{re}}} f\right) \cdot f(x)(\mathrm{mm})$ & $\mathrm{R} 1+\mathrm{W} 1+\mathrm{L} 2=\mathrm{A}(\mathrm{mm})$ & $\mathrm{A} / \lambda$ & $\mathrm{R} 2+\mathrm{W} 3+\mathrm{L} 1=\mathrm{B}(\mathrm{mm})$ & $\mathrm{B} / \lambda$ \\
\hline 1.575 & 152.381 & \multirow{6}{*}{146.85} & $\Lambda$ & \multirow{6}{*}{147.43} & $\lambda$ \\
\hline 2.4 & 100 & & $1.5 \lambda$ & & $1.5 \lambda$ \\
\hline 3.5 & 68.571 & & $2 \lambda$ & & $2 \lambda$ \\
\hline 4.7 & 51.064 & & $2.9 \lambda$ & & $2.9 \lambda$ \\
\hline 5.8 & 41.379 & & $3.5 \lambda$ & & $3.5 \lambda$ \\
\hline 6.5 & 36.923 & & $4 \lambda$ & & $4 \lambda$ \\
\hline
\end{tabular}

relationship of the length can be considered as R1/R2 which is equivalent to one-half wavelength. The result is compared with the total length of the triangular slot on the frequency $2.4 \mathrm{GHz}$ of the desired band. At $3.5 \mathrm{GHz}$, the frequency is almost twice the frequency of $1.575 \mathrm{GHz}$, so the relationship of the length can be considered as L1/L2 which is equivalent to one-half wavelength. The result is compared with the total length of the triangular slot on the frequency $1.575 \mathrm{GHz}$. At 4.7 $\mathrm{GHz}$ and $6.5 \mathrm{GHz}$, the frequencies are almost triple and quadruple the frequency of $1.575 \mathrm{GHz}$, so the relationship of the length can be considered as L1/L2 which is equivalent to three-quarters of the wavelength and full wavelength. At $5.8 \mathrm{GHz}$, the frequency is almost 2.5 times the frequency of $2.4 \mathrm{GHz}$, so the relationship of the length can be considered as $\mathrm{R} 1 / \mathrm{R} 2$ which is equivalent to one-half wavelength. The result is compared with the total length of the triangular slot on the frequency $2.4 \mathrm{GHz}$.

Figure 3 illustrates the final configuration of the proposed high gain and broadband directional antenna for wireless lab applications. There are four stacked layers composed of two antennae and two reflectors, each antenna joints with CPW-feed port, and these two antennas are perpendicular by each other. Etched in the top layer, the antenna serves as the vertical polarization radiation with the $\mathrm{CPW}$-fed in port-1. In the bottom layer, the antenna rotates $90^{\circ}$ to cover the horizontal polarization radiation with the $\mathrm{CPW}$-fed in port-2. To ensure the isolation performance, the distance $\mathrm{D} 1(=15 \mathrm{~mm})$ between the two main antennas should be $0.075 \lambda$ at the desired band of the lowest frequency to reduce the coupling effect. Furthermore, the two reflectors are stacked under the odd function symmetrical dual-polarization equilateral right triangle slot printed broadband directional antenna structure, among the top and bottom antenna layer, and the distance D2 $(=25 \mathrm{~mm})$ and D3 $(=15 \mathrm{~mm})$ should be $0.2 \lambda$ at the desired band of the lowest frequency. The optimized parameter values of the antenna including the reflectors are listed in Table 3.

\section{Experimental Results and Discussion}

To validate the design, the $\mathrm{S}$-parameters and radiation pattern results of the proposed antenna are simulated by using electromagnetic field simulation software Ansys HFSS and CST microwave simultaneously. The antenna prototype has also been fabricated and measured with an eagle view shown in Figure 4.

Figure 5 shows the isolation performance of the two stacked perpendicular odd function symmetrical equilateral right triangle slot with two-fed ports broadband antenna. The parameter D1 is a variable considered to minimize the coupling effect between the two antennas. In the experiment, we observed $\mathrm{D} 1=10 \mathrm{~mm}$ that two input ports' isolation performance simulation result can be achieved $<-17 \mathrm{~dB}$ in the required band, but to minimize the simulation and measured difference, $\mathrm{D} 1=15 \mathrm{~mm}$ is the best distance choice in the final measurement results.

Figures 6-8 show the VSWR and isolation performance of the proposed four-layer odd function symmetrical dualpolarization equilateral right triangle slot printed broadband directional antenna. As a result, the antenna performance VSWR over the HFSS and CST simulation tool has similar curves on port-1 and port-2. There are only two bands impedance mismatch on port-2 in the range of $1.4 \mathrm{GHz} \sim 1.7 \mathrm{GHz}$ and $1.9 \mathrm{GHz} \sim 2.05 \mathrm{GHz}$, and both simulation curves are the same except for the numerical results. To further review the antenna performance that can be achieved in wide impedance bandwidth $\sim 520 \%$ for input return loss $\left|S_{11}\right|$ and output return loss $\left|S_{22}\right|$, VSWR is equal to or smaller than 3 and good isolation between the two input ports $\left|\mathrm{S}_{12}\right|$ is equal or smaller than $-17 \mathrm{~dB}$ for covering $1.37 \mathrm{GHz} 7.12 \mathrm{GHz}$ frequency band. In the parameters D2 and D3, the best spacing needs to be considered by the top and bottom antenna layers for balancing reflector performance and reducing the coupling effect. Hence, D1 + D2 must be equal to $\mathrm{D} 2+\mathrm{D} 3=40 \mathrm{~mm}$ to increase isolation and broadband width in the two antennas. In the experiment, the top antenna layer relies on the 3rd layer reflector to reflect the wave radiation concentrated on $\mathrm{X}$-axis. For the same reason, the bottom antenna layer relies on the 4th layer reflector to reflect most of the wave radiation, including the lower band of the top antenna layer. Note that the shape of third layer reflector is the same as the first layer antenna, that is, for reducing the coupling effect between the second layer and third layer. In addition, the high isolation between the 


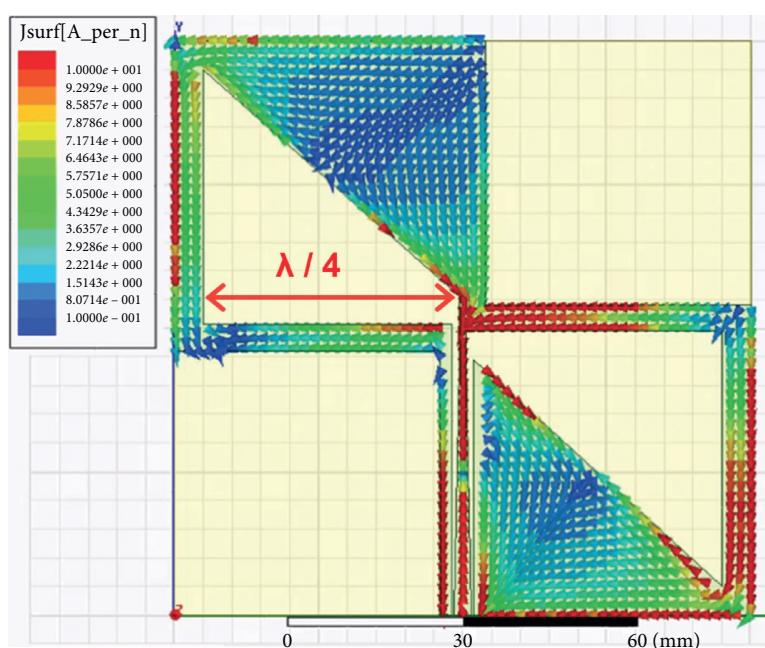

(a)

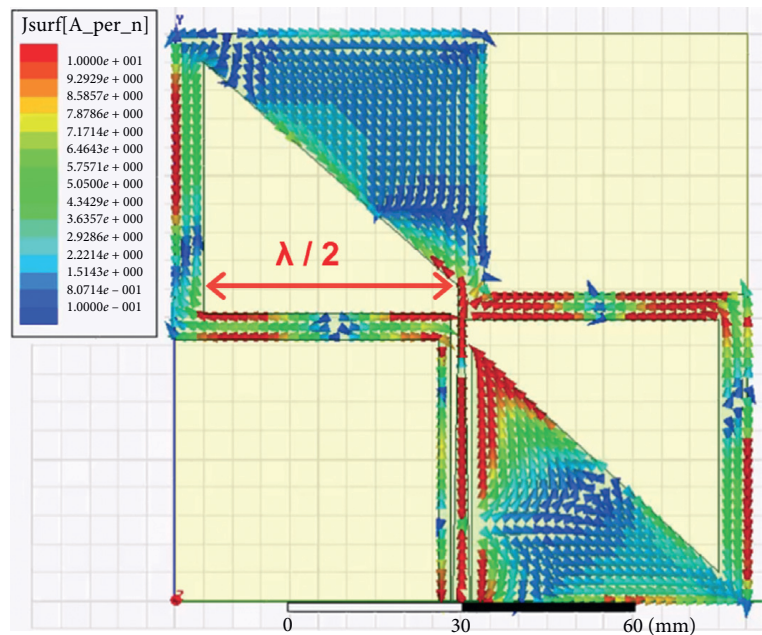

(c)



(e)

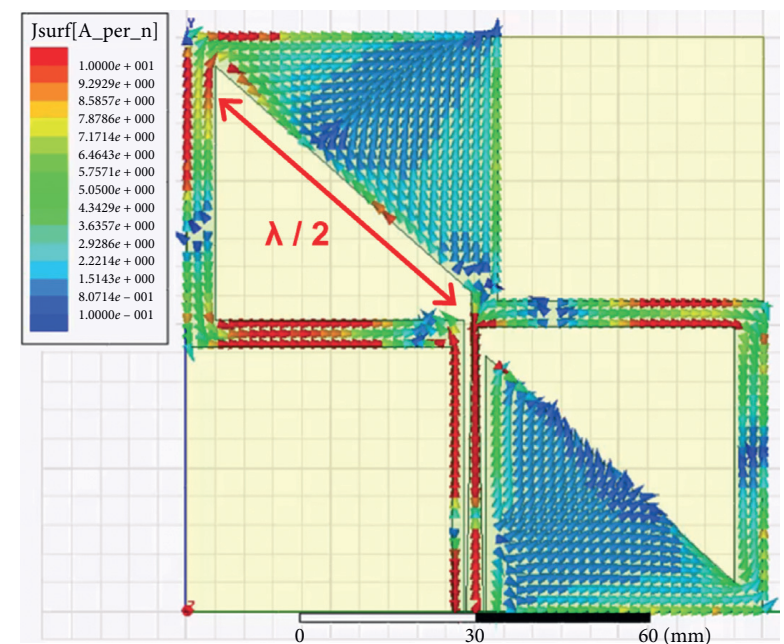

(b)

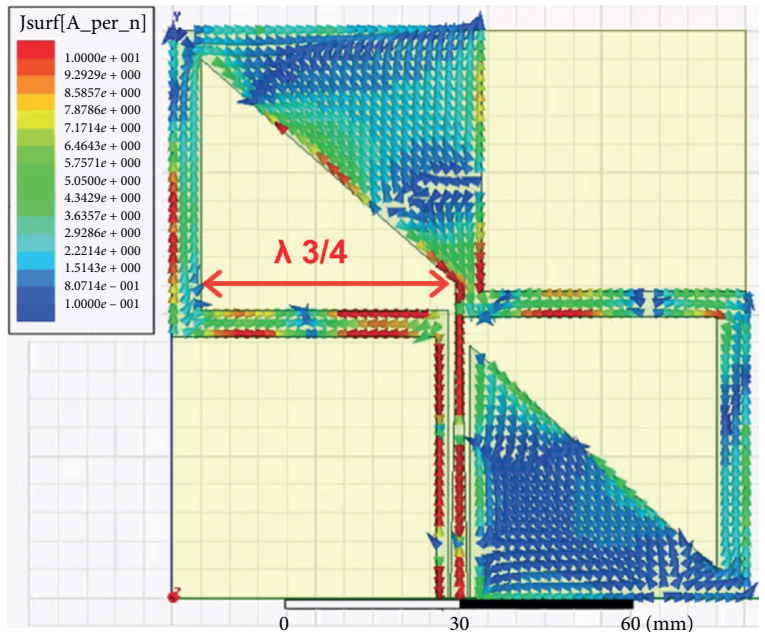

(d)

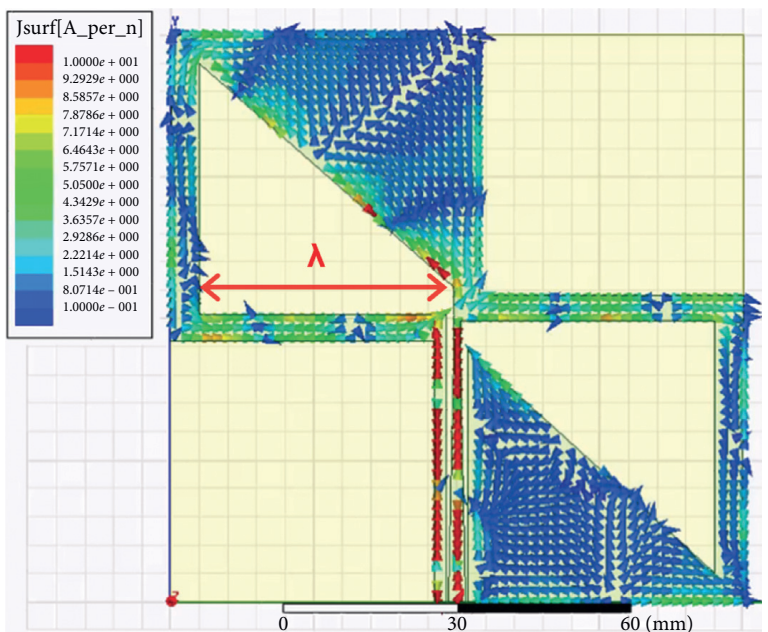

(f)

Figure 2: The relationship between the equivalent current distributions of demand frequency and the fundamental resonance wavelength path. (a) $1.575 \mathrm{GHz}$, (b) $2.400 \mathrm{GHz}$, (c) $3.5 \mathrm{GHz}$, (d) $4.7 \mathrm{GHz}$, (e) $5.8 \mathrm{GHz}$, and (f) $6.5 \mathrm{GHz}$. 


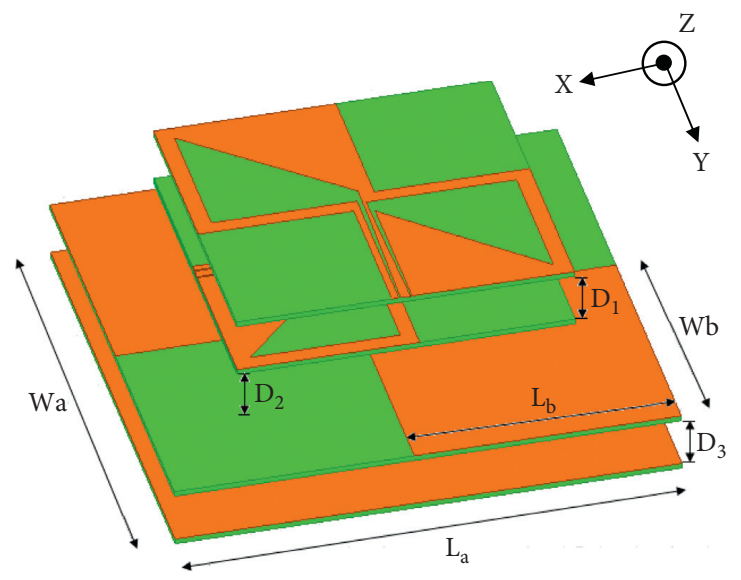

Figure 3: Geometry and dimension of a four-layer odd function symmetrical dual-polarization equilateral right triangle slot printed broadband directional antenna.

TABle 3: Dimensions of a four-layer odd function symmetrical dual-polarization equilateral right triangle slot printed broadband directional antenna.

\begin{tabular}{lccccccc}
\hline Parameter & D1 & D2 & D3 & La & Lb & Wa & Wb \\
\hline Value $(\mathrm{mm})$ & 15 & 25 & 15 & 150 & 79 & 150 & 79 \\
\hline
\end{tabular}

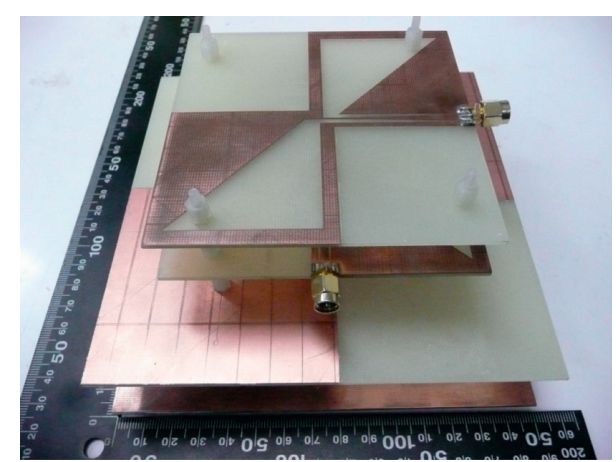

Figure 4: Implemented prototype of the proposed antenna.

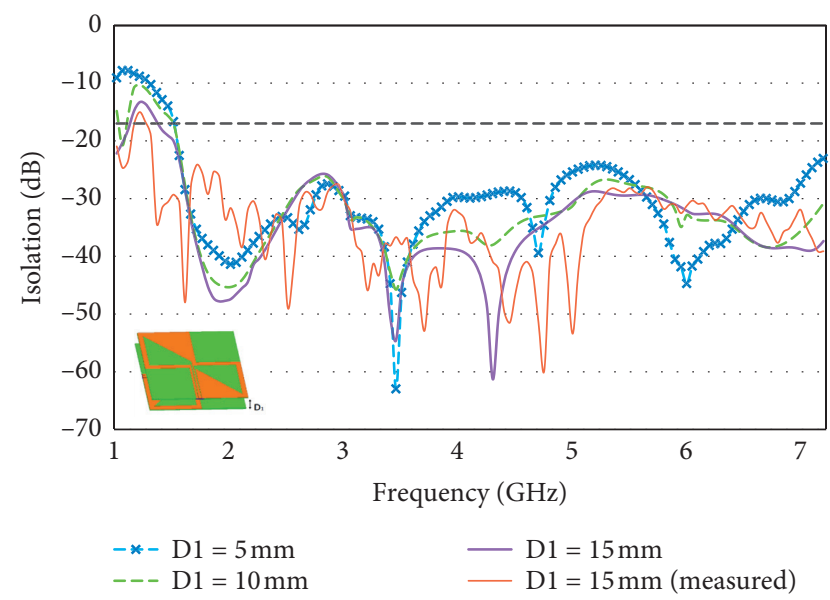

FIGURE 5: Measured and simulated D1 parameter isolation versus frequency. two feeding-ports and the high directional gain are due to the odd function symmetric structure. The parameters $\mathrm{D} 1+\mathrm{D} 2+\mathrm{D} 3=55 \mathrm{~mm}$ also meet the definition of the antenna reflector theory; that is, the gap distance between antennas must be greater than one-sixth $\lambda$ by the lowest frequency of the desired band. It is demonstrated that the coupling effect can be minimized and the reflector performance has been optimized for top and bottom antenna.

Figure 9 presents the (i) VSWR and $\left|S_{12}\right|$ experimental measurement photo of the antenna under test (AUT), which is measured with Copper Mountain Technologies C4209 Vector Network Analyzer (VNA). The (ii) 3D radiation pattern measurement was implemented by the 3D anechoic chamber of ETS-Lindgren.

The proposed antenna supporting dual-polarization is the primary purpose, so the $2 \mathrm{D}$ radiation pattern of the $\mathrm{xz}$ plane at port- 1 and yz-plane at port- 2 should be similar to what is expected. When feeding on port- 1 and port- 2 , the radiation patterns for free space of the proposed antenna are shown in Figures 10-12. For port-1, the vertical polarization is the dominant polarization. The half-power ( $3 \mathrm{~dB}$ ) beamwidths are $60^{\circ}$ and $40^{\circ}$ in E1-plane (xz-plane) and E2-plane (yz-plane), and the maximum radiation power (MRP) is concentrated at about $0^{\circ}$ on the plus $\mathrm{Z}$-axis at $1.575 \mathrm{GHz}$. For port-2, the horizontal polarization is the dominant polarization. The $3 \mathrm{~dB}$ beamwidths are $40^{\circ}$ and $40^{\circ}$ in $x z$-plane and yz-plane, and the MRP is concentrated on the same axis as port- 1 at $1.575 \mathrm{GHz}$. At a frequency of $2.4 \mathrm{GHz}$, the $3 \mathrm{~dB}$ beamwidths are $60^{\circ}$ in $\mathrm{xz}$ plane and yz-plane in port- 1 and $50^{\circ}$ in $x z$ - and yz-plane in port-2, and the MRP of the two ports are the same and they are concentrated at about $0^{\circ}$ on the plus $\mathrm{Z}$-axis. From the perspective of $5 \mathrm{G}$ and higher frequency radiation patterns, when the operating frequency is higher than $5 \mathrm{GHz}$, the $3 \mathrm{~dB}$ beamwidths are almost limited to around $20^{\circ}$ in $\mathrm{xz}$-plane and yz-plane, and the MRP is scattering more seriously because the wavelength of $5 \mathrm{G}$ and higher frequencies has tripled relative to the lowest frequency of the desired band. 




FIGURE 6: Measured and simulated VSWR (port-1) versus frequency of the proposed antenna.

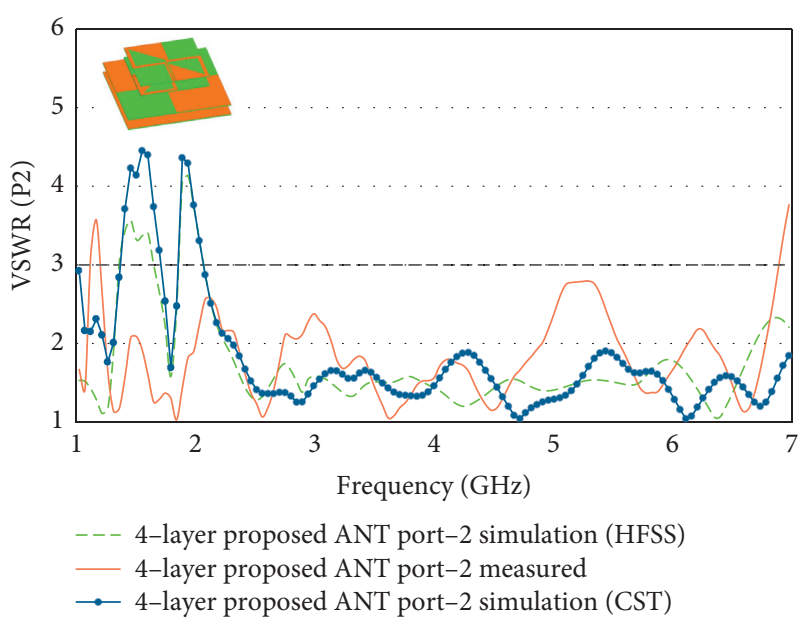

FIGURE 7: Measured and simulated VSWR (port-2) versus frequency of the proposed antenna.

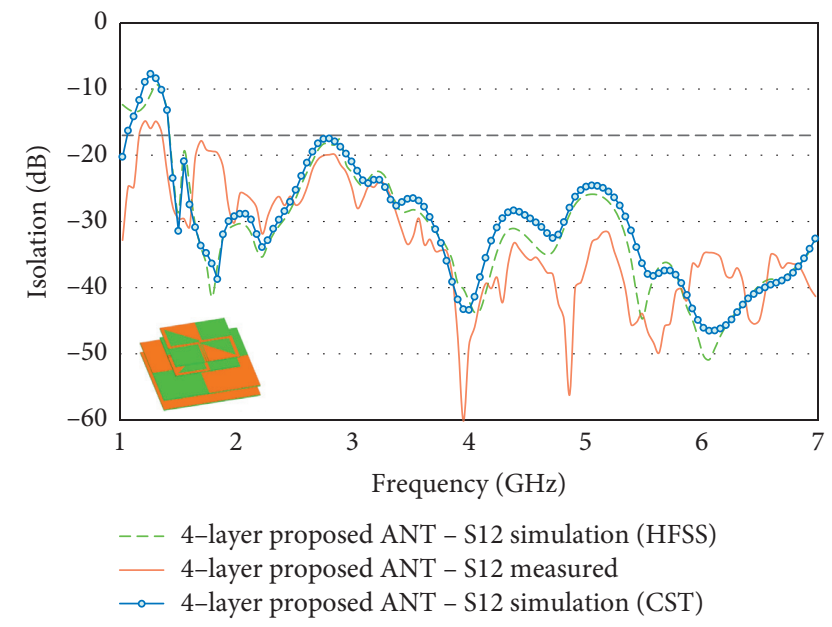

FIGURE 8: Measured and simulated isolation versus frequency of the proposed antenna.
To summarize the measured results of all the radiation patterns in xz-plane and yz-plane, the proposed antenna radiation pattern is similar when feeding port- 1 and port- 2 . The phase difference of $90^{\circ}$ between the two antennas will make the radiation pattern have obvious polarization diversity. By analyzing in E-plane results, it is proved that the odd symmetrical antenna has obtained both polarization diversity and pattern diversity characteristics with good isolation.

The 3D pattern efficiency and peak gain of the proposed antenna are also measured. The measurement result was done by using pattern integration employing the ETSLindgren anechoic chamber and it is shown in Figure 13. In the GPS band of $1.575 \mathrm{GHz}$, the efficiency and peak gain is $61.4 \%$ and $5.2 \mathrm{dBi}$ for port- 1 and $58.6 \%$ and $5.8 \mathrm{dBi}$ for port2. In the WLAN band of $2.4 \mathrm{GHz}, 5.8 \mathrm{GHz}$, and $6.5 \mathrm{GHz}$, the efficiencies are $69.3 \%, 69.1 \%$, and $63.6 \%$ for port-1 and $72.6 \%, 70 \%$, and $57 \%$ for port- 2 . The peak gain is $5.6,7.1$, and $5.3 \mathrm{dBi}$ for port- 1 and $5.3,6$, and $4.6 \mathrm{dBi}$ for port- 2 . In the sub- $65 \mathrm{G}$ band of 3.5 and $4.7 \mathrm{GHz}$, the efficiencies are $52 \%$ and $76 \%$ for port -1 and $52.5 \%$ and $75 \%$ for port- 2 . The peak gain is 4.2 and $7.7 \mathrm{dBi}$ for port- 1 and 5 and $6.5 \mathrm{dBi}$ for port- 2 .

In Figure 12, it was found that the radiation patterns are multilobe. This is probably because, for higher frequencies in the upper-frequency band, some undesired higher-order modes of the multilayer structure are excited, which could cause some distortions in the resultant radiation patterns. Based on the radiated pattern requirements of wireless consumer products, too many distorted radiation patterns are not convincing. But the proposed antenna is a two-port dual-polarization antenna for measurement applications. As long as one of the two planes $\mathrm{y}-\mathrm{z}$ and $\mathrm{z}$ - $\mathrm{x}$ has the peak gain of the antenna, it can transmit and receive power, which meets the requirements of measurement antenna in the testing laboratory [14].

Table 4 displays the comparison among the proposed antenna and other wideband antennas. Most of the reference antennas have a wider FBW (fractional bandwidth, FBW) of around $85.7 \%(2-5 \mathrm{GHz})$ with dual-polarization 


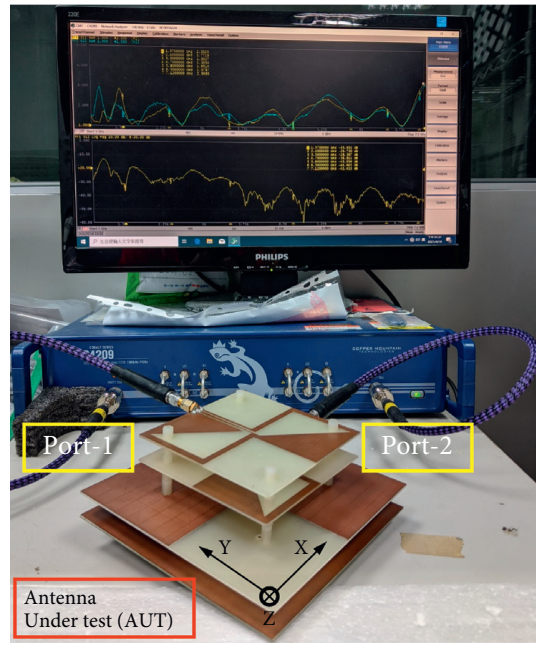

(a)



(b)

FIgURE 9: Photo of experimental measurement for (a) VSWR and $\left|S_{12}\right|$ and (b) 3D radiation pattern of the proposed antenna.

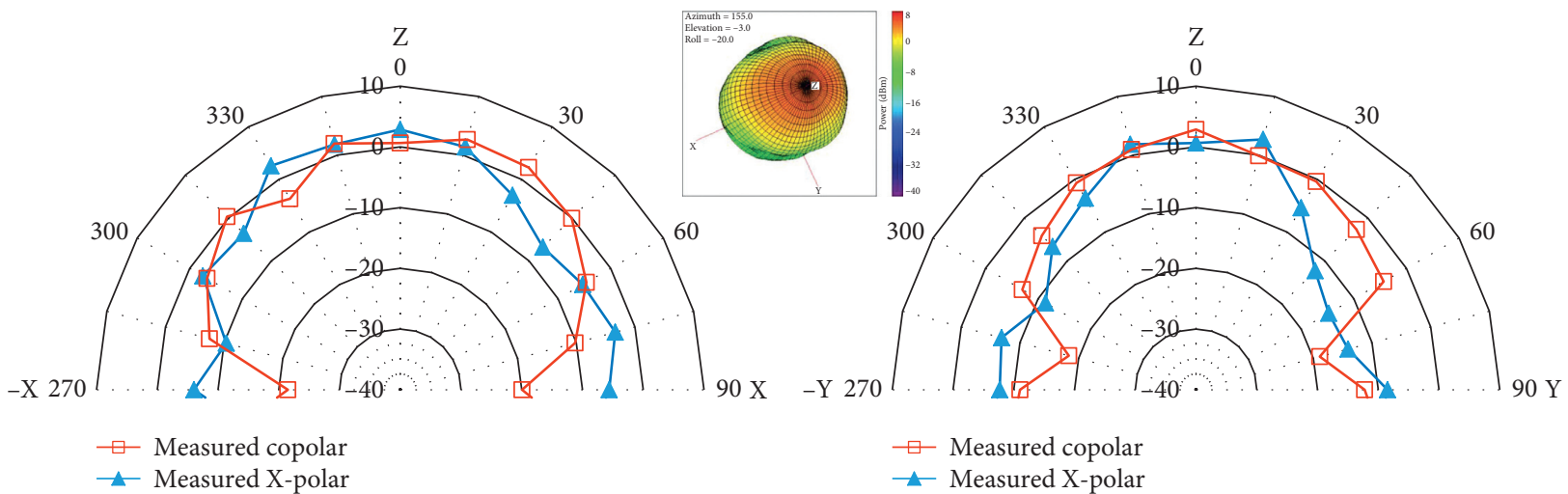

(a)
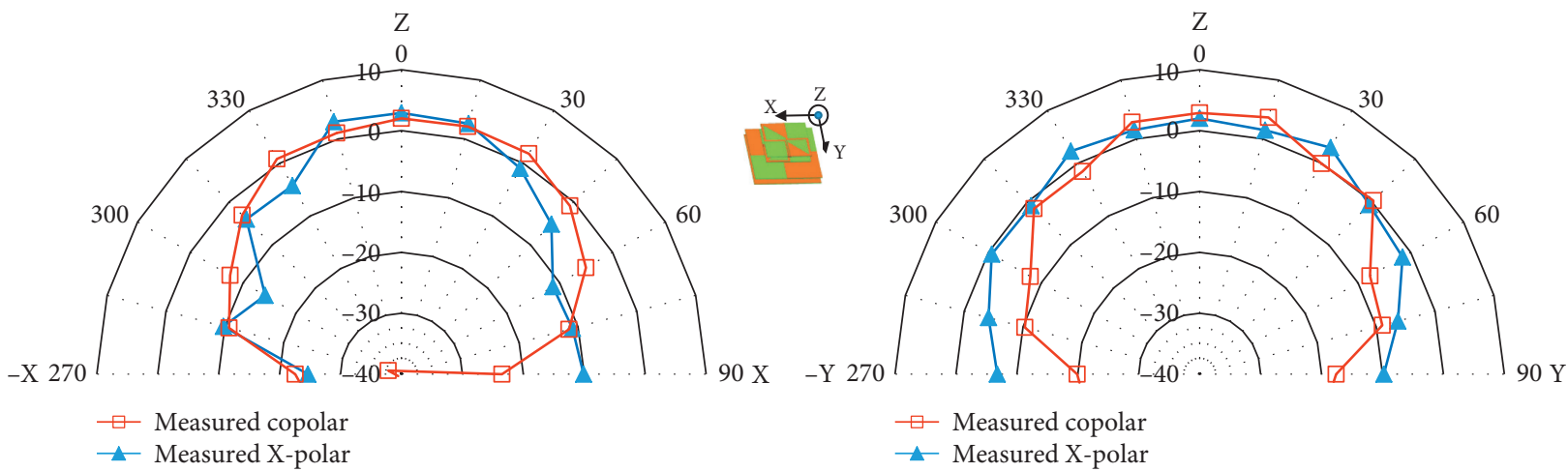

(b)

FIGURE 10: Measured radiation patterns when feeding on xz-plane and yz-plane at $1.575 \mathrm{GHz}$ (a) port-1 and (b) port-2.

and directional radiation. For mobile base station or lab measurement applications, the antenna in [9] does not support dual-polarization, even if $[10-13,15]$ have better antenna gain in the frequency range of $1-6 \mathrm{GHz}$, but these antennas have insufficient bandwidth to cover modern wireless technologies and require extra feeding network design and expensive materials. Different from
$[10-13,15]$, the proposed antenna utilizes an odd function symmetrical equilateral right triangle slot to realize an ultrawide bandwidth of $1.37-7.12 \mathrm{GHz}(135.5 \%)$. It can be seen that the proposed antenna has the advantages of ultrawide bandwidth, low cost, planar and simple structure, and good enough performance in isolation and antenna gain. 


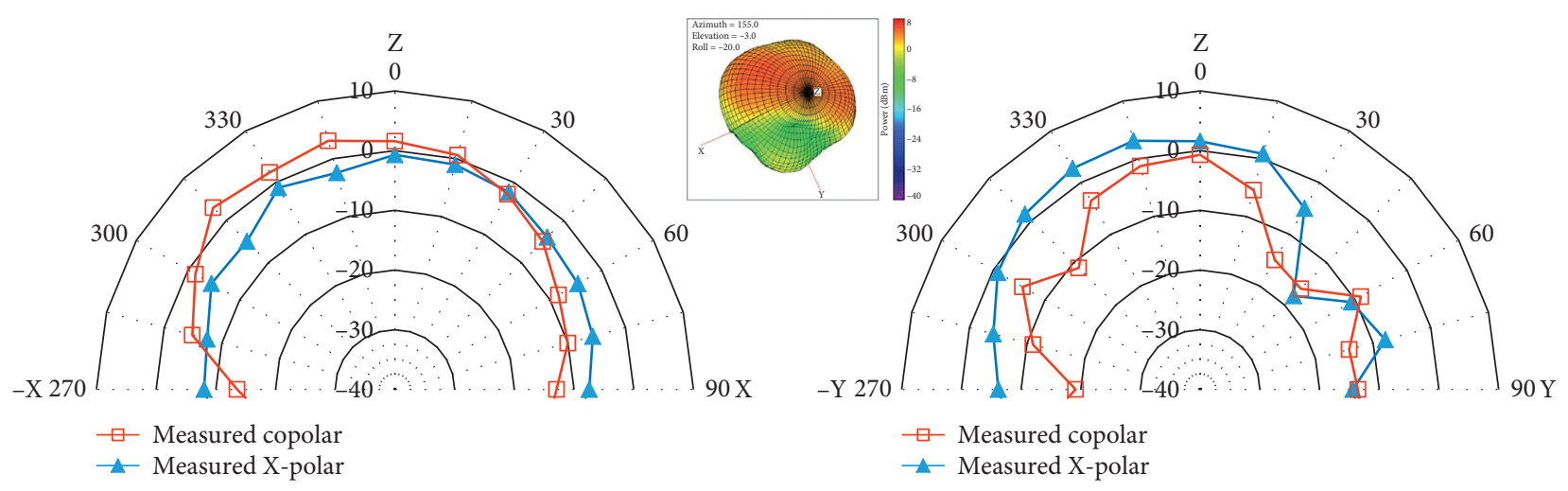

(a)
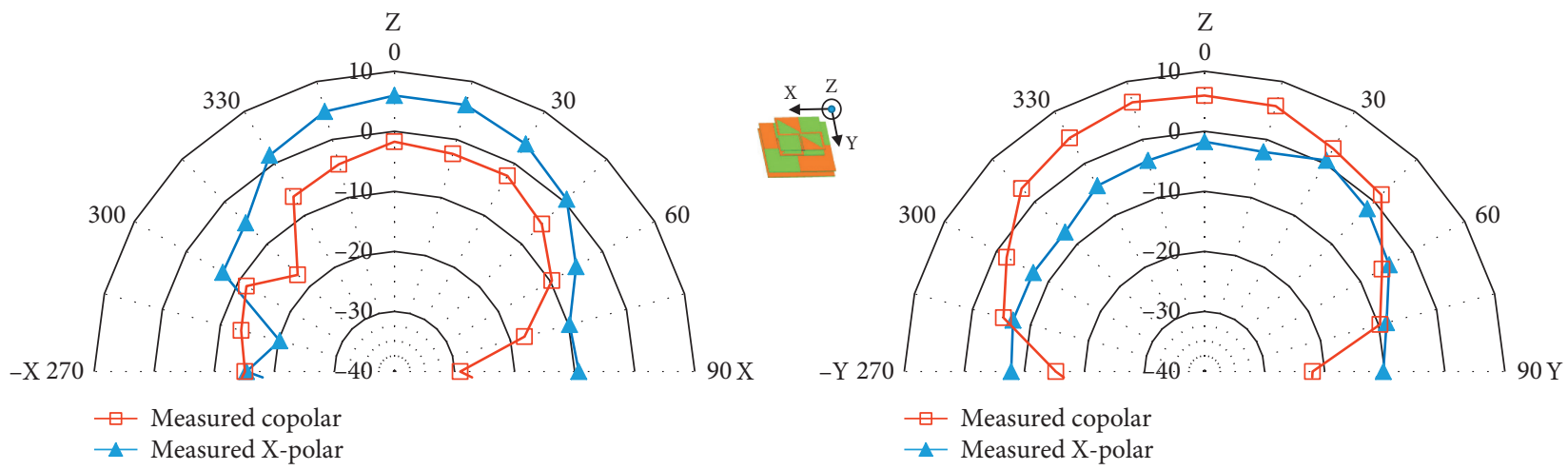

(b)

Figure 11: Measured radiation patterns when feeding on xz-plane and yz-plane at $2.4 \mathrm{GHz}$ (a) port-1 and (b) port-2.

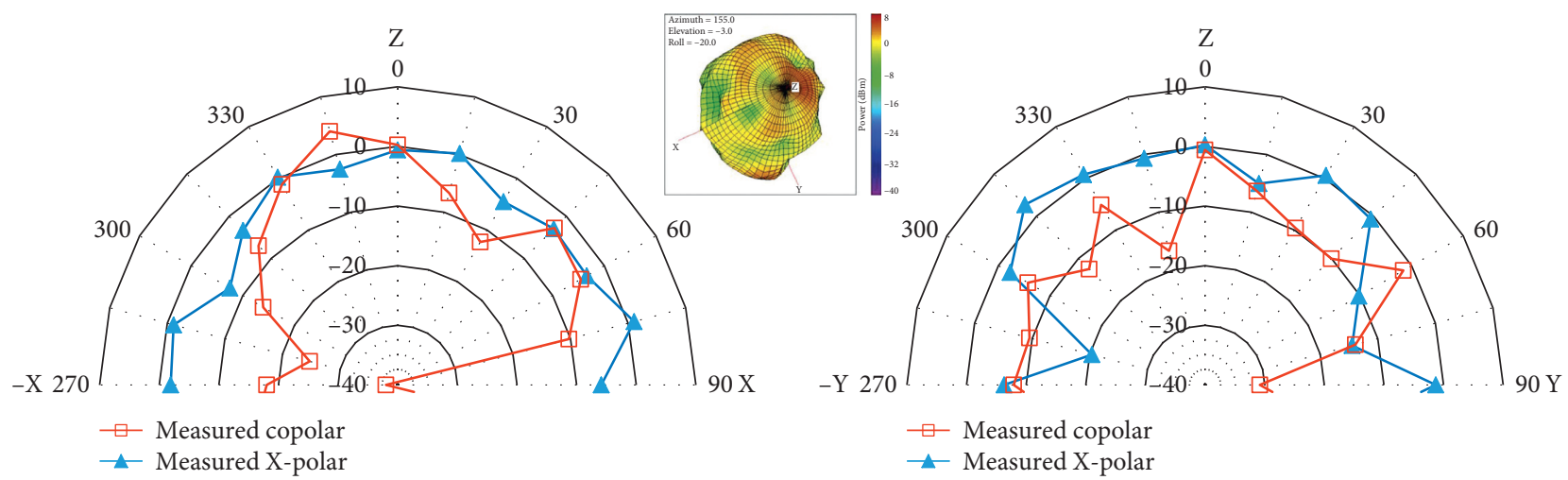

(a)
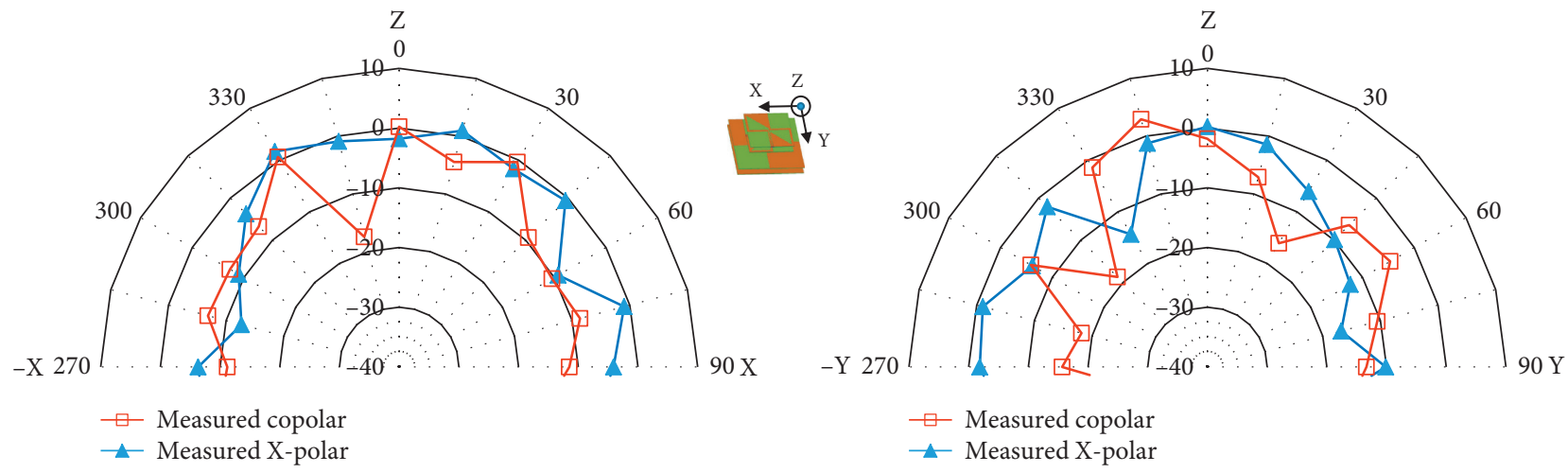

(b)

Figure 12: Measured radiation patterns when feeding on xz-plane and yz-plane at $5.8 \mathrm{GHz}$ (a) port-1 and (b) port-2. 


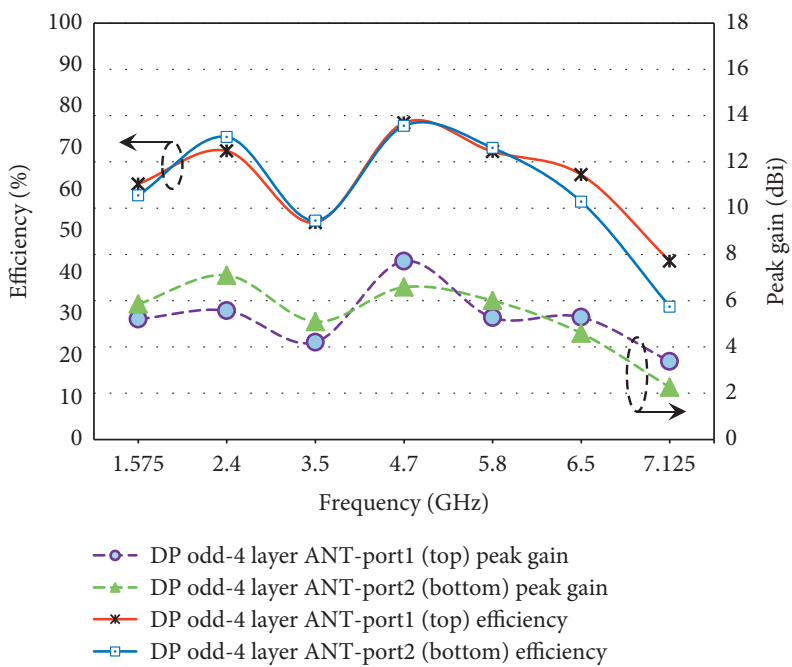

FIGURE 13: Measured antenna peak gain and efficiency versus frequency of the proposed antenna.

TABLE 4: Comparison of different antennas.

\begin{tabular}{|c|c|c|c|c|c|c|c|}
\hline Refs. & Size $\left(\mathrm{mm}^{3}\right)$ & $\begin{array}{c}\text { Bandwidth (BW/ } \\
\text { GHz) }\end{array}$ & Isolation $(\mathrm{dB})$ & Peak gain $(\mathrm{dBi})$ & Dual polar & $\begin{array}{l}\text { Extra feeding } \\
\text { network }\end{array}$ & Materials \\
\hline $\begin{array}{l}\text { This } \\
\text { work }\end{array}$ & $150 \times 150 \times 62$ & $135.5 \%(1.37 \sim 7.12)$ & $\leqq-17$ & $3.4-7$ & Yes & No & FR4 \\
\hline [9] & $240 \times 144 \times 132$ & $\begin{array}{c}36.7 \%(0.69 \sim 1), 47.3 \% \\
(1.71 \sim 2.77)\end{array}$ & NA & $4-6$ & No & No & Teflon \\
\hline$[10]$ & $90 \times 90 \times 23$ & $121.4 \%(2.69 \sim 11)$ & $\leqq-28$ & $3.9-10$ & Yes & Yes & $\begin{array}{c}\text { Rohacell } \\
\text { foam + FR } 4+\text { copper }\end{array}$ \\
\hline$[11]$ & $160 \times 160 \times 1$ & $55.9 \%(2.4 \sim 4.26)$ & $\leqq-35$ & $6-8.4$ & Yes & Yes & Rogers RT/duroid 5880 \\
\hline$[12]$ & $140 \times 140 \times 31.2$ & $70 \%(1.5 \sim 3.1)$ & $\leqq-35$ & $7.2-9.5$ & Yes & Yes & FR4 \\
\hline$[13]$ & $70 \times 70 \times 20.5$ & $91.8 \%(2.38 \sim 6.42)$ & $\leqq-31$ & $6.3-9.5$ & Yes & No & Rogers 4350 \\
\hline$[15]$ & $130 \times 130 \times 45$ & $94.8 \%(0.91 \sim 2.55)$ & $\leqq-20$ & $4.5-8$ & Yes & No & Metallic plates \\
\hline
\end{tabular}

This work: VSWR $\leqq 3 ;[9-13,15]:$ VSWR $\leqq 2$.

\section{Conclusion}

A four-layer odd function symmetrical dual-polarization equilateral right triangle slot printed broadband directional antenna for wireless lab measurement applications has been demonstrated and measured. The antenna structure is comprised of the two equilateral right triangle slots with odd function symmetrical pair of stubs; there are four stacked layers composed of two antennae and two reflectors, each antenna joints with CPW-feed port, and these two antennas are perpendicular by each other. By correctly choosing the length and width of the slots, it can obtain much wider impedance bandwidth, dual-polarization, and high isolation. The measured results demonstrate that the proposed antenna performance can achieve a wide impedance bandwidth $\sim 520 \%$ for $\left|S_{11}\right|$ and $\left|S_{22}\right|$; VSWR $\leqq 3$ which has implemented the operating band from 1.37 to $7.12 \mathrm{GHz}$ for GPS (1.575 GHz), WLAN band $(2.4 \mathrm{GHz}$ and $5.8 \mathrm{GHz})$, and $6 \mathrm{GHz}$ unlicensed spectrum $(5.925 \mathrm{GHz} 7.125 \mathrm{GHz})$, Long Term Evolution Upper band (LTE, 1710-2690 MHz), and sub-6 $5 \mathrm{G}$ band $(3.5 \mathrm{GHz}$ and $4.7 \mathrm{GHz})$ applications. The measured inband isolation performance between the two input ports is valuable for achieving $\left|S_{12}\right| \leqq-17 \mathrm{~dB}$. Although the original antenna specification was designed for frequency $1.5 \mathrm{GHz}-7.125 \mathrm{GHz}$ applications, the design concept can also be extended to other frequency bands of interest.

\section{Data Availability}

The data used to support the findings of this study are included within the article.

\section{Conflicts of Interest}

The authors declare that they have no conflicts of interest.

\section{Acknowledgments}

The authors thank the project for technical R\&D and service team of Dong Guan Polytechnic, Intelligent Terminal Product R\&D and Technical Service Team (no. CXTD201802). This work was sponsored by Wieson Corp. Tech. (http://www. wieson.com), Taiwan, under the Contract JW109-F-315-018. 


\section{References}

[1] H. Zhong, Z. Zhang, W. Chen, Z. Feng, and M. F. Iskander, "A tri-polarization antenna fed by proximity coupling and probe," IEEE Antennas and Wireless Propagation Letters, vol. 8, pp. 465-467, 2009.

[2] C.-H. Lee, S.-Y. Chen, and P. Hsu, "Isosceles triangular slot antenna for broadband dual polarization applications," IEEE Transactions on Antennas Propagation, vol. 57, no. 10, pp. 3347-3351, 2009.

[3] Y. Li, Z. Zhang, W. Chen, Z. Feng, and M. F. Iskander, "A dual-polarization slot antenna using a compact CPW feeding structure," IEEE Antennas and Wireless Propagation Letters, vol. 9, pp. 191-194, 2010.

[4] C. Y. Huang and D. Y. Lin, "CPW-fed bow-tie slot antenna for ultrawideband communications," Electronics Letters, vol. 42, no. 19, pp. 1073-1074, 2006.

[5] I.-F. Chen, C.-M. Peng, C.-C. Hung, and H.-C. Chen, "Printed modified bow-tie dipole antenna for multi-band appications," IEICE Transactions on Communications, vol. E92-B, no. 4, pp. 1404-1405, 2009.

[6] C.-C. Hung, C.-M. Peng, and I.-F. Chen, "Printed modified bow-tie dipole antenna for DVB/WLAN applications," Hindawi International Journal of Antennas and Propagation, vol. 2013, Article ID 149296, 6 pages, 2013.

[7] M.-T. Wu and M.-L. Chuang, "Multibroadband slotted bowtie monopole antenna," IEEE Antennas and Wireless Propagation Letters, vol. 14, pp. 887-890, 2015.

[8] C.-J. Wu, I.-F. Chen, C.-M. Peng, and J.-S. Sun, "A dual polarization bow-tie slot antenna for broadband communications," The International Journal of Engineering and Science (IJES), vol. 7, no. 6, pp. 49-56, 2018.

[9] X. Z. Zhu, J. L. Zhang, T. Cui, and Z. Q. Zheng, "A dualbroadband printed dipole antenna for $2 \mathrm{G} / 3 \mathrm{G} / 4 \mathrm{G}$ base station applications," Hindawi International Journal of Antennas and Propagation, vol. 2019, Article ID 4345819, 7 pages, 2019.

[10] F. G. Zhu, S. Gao, A. T. S. Ho et al., "Ultra-wideband dualpolarized patch antenna with four capacitively coupled feeds," IEEE Transactions on Antennas Propagation, vol. 62, no. 5, pp. 2440-2449, 2014.

[11] C. Wu, C. Lu, and W. Cao, "Wideband dual-polarization slot antenna with high isolation by using microstrip line balun feed," IEEE Antennas and Wireless Propagation Letters, vol. 16, pp. 1759-1762, 2017.

[12] K. Sun, D. Yang, Y. Chen, and S. Liu, "A broadband commonly fed dual-polarized antenna," IEEE Antennas and Wireless Propagation Letters, vol. 17, no. 5, pp. 747-750, 2018.

[13] Y. Zhang, Y. Zhang, D. Li, K. Liu, and Y. Fan, "Ultrawideband dual-polarized antenna with three resonant modes for $2 \mathrm{G} / 3 \mathrm{G} / 4 \mathrm{G} / 5 \mathrm{G}$ communication systems," IEEE Access, vol. 7, pp. 43214-43221, 2019.

[14] W. Yang and Y. Pan, "A wideband dual-polarized dipole antenna with folded metallic plates," IEEE Antennas and Wireless Propagation Letters, vol. 17, no. 10, pp. 1797-1801, 2018.

[15] IEEE Standards Board, "IEEE standard test procedures for antennas," in ANSI/IEEE Std 149-1979, IEEE Standards Board, New York, NY, USA, 1979. 